$\underset{\text { publications }}{\mathbf{A}} \underset{\mathbf{C}}{\mathbf{C}} \mathbf{G}$

Org. Commun. 14:3 (2021) 228-239 organic communications

\title{
The use of crown ethers as sensor material in potentiometry
}

\section{technique}

\author{
Oguz Özbek ${ }^{1}{ }^{1, *}$, Ömer Isildak $\oplus^{2}$, Meliha Burcu Gürdere $\bigodot^{2}$ \\ and Alper Cetin ${ }^{3} 3$
}

\author{
${ }^{1}$ Science and Technology, Application and Research Center, Zonguldak Bulent Ecevit \\ University, 67600 Zonguldak, Türkiye \\ ${ }^{2}$ Department of Chemistry, Faculty of Science and Arts, Tokat Gaziosmanpasa University, \\ 60250 Tokat, Türkiye \\ ${ }^{3}$ Department of Molecular Biology and Genetics, Faculty of Science and Arts, Zonguldak \\ Bulent Ecevit University, 67100 Zonguldak, Türkiye
}

(Received June 25, 2021; Revised August 07, 2021; Accepted August 12, 2021)

\begin{abstract}
Potentiometric methods are a type of electrochemical analysis which are used widely in many applications due to their multiple advantages such as wide concentration range, low detection limit, high selectivity, and sensitivity. Potentiometric sensors have many advantages over the other analytical methods and have been successfully applied in different real sample analyzes. Crown ethers are a group of macrocyclic compounds and have been used as ionophores by researchers due to their favorable chemical structures. In this review, we provide a description on crown ethers used as ionophores in potentiometric sensors.
\end{abstract}

Keywords: Crown ether; ion-selective electrode; potentiometry; ionophore; sensor. C2021 ACG Publication. All right reserved.

\section{Introduction}

Synthesis of crown ether compounds is important in organic and supramolecular chemistry. ${ }^{1}$ The first synthetic method was reported by Charles Pedersen in 1967 (Figure 1) ${ }^{2,3}$ Crown ethers contain multiple $-\mathrm{CH}_{2} \mathrm{CH}_{2} \mathrm{O}$ - units, which are connected to form a circular molecule. ${ }^{4}$ They have advantages of high resistance to chemicals, temperature, radiolysis, and polar solvents. ${ }^{5}$ One of their most important features is that they can make complexes with a wide variety of cations in the empty cavity center in the center of the ring (Figure 2) ${ }^{6,7}$ They have attracted attention as their unique capability to form hostguest (1:1) complexes with cations. ${ }^{8}$ The stability of the host-guest complex depends on the number of crown ether donor atoms. ${ }^{9}$ They have been used in many fields, including chemical sensors, organic synthesis, chromatographic techniques, and phase transfer catalysts. ${ }^{10}$ In addition, they are known to exhibit various biological activities such as antimicrobial, ${ }^{11}$ antiproliferative, ${ }^{12}$ antifungal, ${ }^{13}$ antiinflammatory ${ }^{14}$ and antimutagenic. ${ }^{15}$

\footnotetext{
*Corresponding author: E-Mail: oguz.ozbek@ beun.edu.tr, Phone: + 903722613285. 
Özbek et al., Org. Commun. (2021) 14:3 228-239

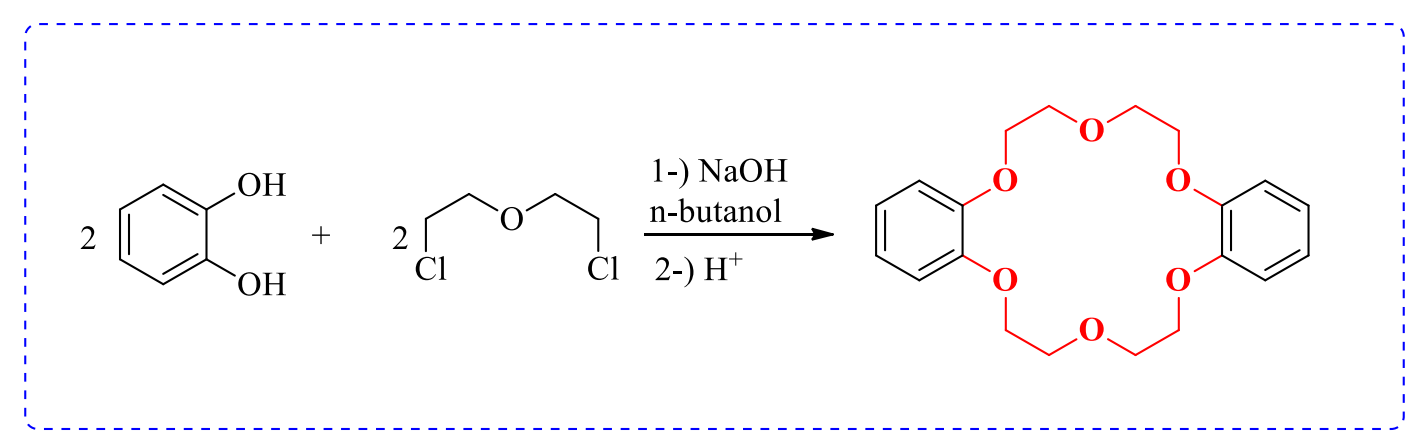

Figure 1. Pedersen's synthesis of first crown ether

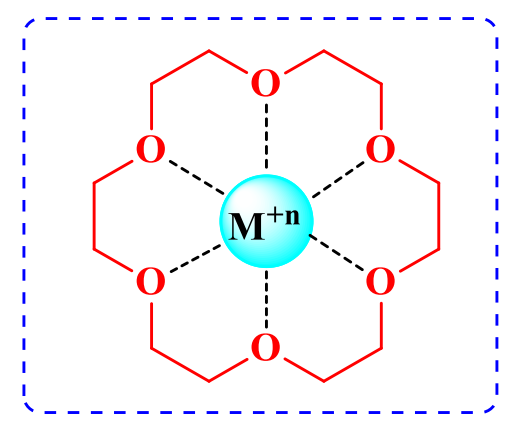

Figure 2. The metal complex of the crown ethers

Potentiometric methods are highly important in the field of electroanalytical chemistry and commonly used for the determination of different ions. ${ }^{16,17}$ Potentiometric ion-selective electrodes offer important advantages including wide concentration range, low detection limit, long lifetime, low cost, ease of use, high selectivity and sensitivity and short response time etc. ${ }^{18-22}$ Because of these advantages, potentiometric sensors are widely used in areas such as environmental monitoring, industrial, agricultural and drug analysis. ${ }^{23,24}$ A significant number of crown ether derivatives have been used in the construction of potentiometric sensors to determine different metal cations in real samples.

Ionophore, the most important component of potentiometric ion-selective electrodes (ISEs) is responsible for the selective response to a target ion. ${ }^{25,26}$ Macrocyclic compounds, including porphyrins, crown ethers and calixarenes are often used as materials in potentiometry-based sensors due to their sensitivity and selectivity toward various ions.

\section{Crown-Ether Based Potentiometric Sensors}

Crown ether-based potentiometric sensors have shown significant sensitivity to many different cations. Gupta et al. developed an aluminum(III)-selective potentiometric electrode using 12-crown-4 (1) (Figure 3) as ionophore. ${ }^{27}$ This aluminum(III)-selective electrode in the linear range of $1.0 \times 10^{-6}$ $1.0 \times 10^{-1} \mathrm{M}$, did not affect the changes in $\mathrm{pH}$ between 2.0-7.8. In addition, the developed electrode was applied for the analysis of aluminum(III) in andesite, basalt, rhyolite, granite, and Al-Mg syrup, and results were given in comparison to atomic emission spectrometry (AES). Another work, where a 12crown-4 (1) molecule was used as an ionophore in the structure of zinc(II)-selective sensor, was proposed by Gupta. ${ }^{28}$ The proposed potentiometric sensor had a wide concentration range of $7.0 \times 10^{-5}$ $-1.0 \times 10^{-1} \mathrm{M}$ against $\mathrm{Zn}^{2+}$ ions and a response time of less than $10 \mathrm{~s}$. Kumar et al. reported a copper(II)selective potentiometric sensor using the same ionophore (1) ${ }^{29}$ The developed sensor worked in a concentration range of $1.78 \times 10^{-5}-1.0 \times 10^{-1} \mathrm{M}$ and had a response time of $<30 \mathrm{~s}$.

Khaled et al. developed a disposable screen-printed lead(II)-selective potentiometric sensor using 15-crown-5 (2) (Figure 3) as an ionophore. ${ }^{30}$ They reported that this sensor worked in the linear concentration range of $1.0 \times 10^{-6}-1.0 \times 10^{-2} \mathrm{M}$ and had a lower detection limit of $2.0 \times 10^{-7} \mathrm{M}$. This lead(II)-selective sensor had a response time of $<2 \mathrm{~s}$, and was successfully applied to the determination of $\mathrm{Pb}^{2+}$ ions in environmental samples. Another potentiometry-based sensor developed by Karimian et 
The use of crown ethers as sensor material in potentiometry technique

al. was a silver(I)-selective sensor prepared by using 15 -crown-5 (2) as an ionophore, ${ }^{31}$ which was reported to have a wide working concentration range of $1.0 \times 10^{-7}-1.0 \times 10^{-1} \mathrm{M}$. It had a lifetime of 2 months and a response time of approximately $10 \mathrm{~s}$. The proposed sensor was successfully used in the determination of $\mathrm{Ag}^{+}$ions in radiology wastes. They compared the results of the sensor $(2.77 \pm 0.04$ mmol L-1) with atomic absorption spectrometry (AAS) $\left(2.65 \pm 0.02 \mathrm{mmol} \mathrm{L}^{-1}\right)$, and reported to be in a good agreement.

A study on lead(II)-selective coated graphite potentiometric electrode using a benzo-18-crown6 (3) was performed by Ghorbani et al. ${ }^{32}$ The sensor had a Nernstian slope of $28.80 \mathrm{mV} /$ decade and a $\mathrm{pH}$ working range of 1.5 to 5.0. Ekmekci et al. developed an iron(III)-selective potentiometric electrode with the same ionophore (3) (Figure 3) ${ }^{33}$ The electrode worked in a wide concentration range of $1.0 \times$ $10^{-6}-1.0 \times 10^{-1} \mathrm{M}$, and was successfully applied in blood and grape molasses. Ganjali et al. reported a poly(vinly chloride) (PVC) membrane beryllium selective sensor using napto-9-crown-3 (4) (Figure 3) as an ionophore. ${ }^{34}$ The beryllium selective sensor showed a wide concentration range of $8.0 \times 10^{-6}-1.0$ $\times 10^{-1} \mathrm{M}$ with a detection limit of $6.0 \times 10^{-6} \mathrm{M}$. In addition, this sensor was successfully applied to the determination of $\mathrm{Be}^{2+}$ ions in binary mixtures. Govindan et al. reported a potentiometric lithiumselective electrode using 6,6-dibenzyl-14-crown-4 (5) (Figure 3) as an ionophore. ${ }^{35}$ The electrode was successfully applied to the determination of lithium ions in complex chemical matrices.

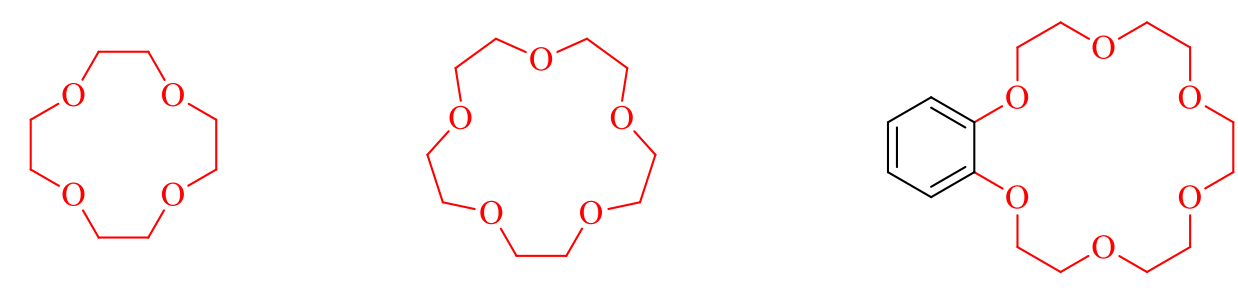

12-crown-4 (1) 15-crown-5 (2) benzo-18-crown-6 (3)<smiles>c1ccc2cc3c(cc2c1)OCCOCCO3</smiles><smiles>c1ccc(CC2(Cc3ccccc3)COCCCOCCOCCCO2)cc1</smiles>

naphto-9-crown-3 (4)

6,6-dibenzyl-14-crown-4 (5)

Figure 3. Crown ethers 1, 2, 3, 4 and 5 used as ionophores

Gupta and coworkers used dicyclohexano-18-crown-6 (6) (Figure 4) as an ionophore to fabricate a cadmium(II)-selective sensor, ${ }^{36}$ which worked at a concentration fange of $2.1 \times 10^{-5}-1.0 \times$ $10^{-1} \mathrm{M}$ and had a Nernstian slope of $29.0 \pm 1.0 \mathrm{mV} /$ decade. The proposed sensor was applied in wastewater samples. Mittal et al. used the same molecule (6) (Figure 4) as an ionophore in the lanthanum(III)-selective sensor. ${ }^{37}$ The sensor was reported to have a working range of $1.0 \times 10^{-6}-1.0$ $\times 10^{-1} \mathrm{M}$ and had a low detection limit $\left(5.0 \times 10^{-7} \mathrm{M}\right)$. In addition, the lanthanum(III)-selective sensor was not affected by the change of $\mathrm{pH}$ in the range of 4.0-9.0.

PVC membrane cadmium(II)-selective sensor was developed by Gupta et al. using dicyclohexano-24-crown-8 (Figure 4) (7) as an ionophore. ${ }^{38}$ This sensor had a working range of $3.0 \times$ $10^{-5}-1.0 \times 10^{-1} \mathrm{M}$ and exhibited Nernstian behavior (30.0 $\pm 1.0 \mathrm{mV} /$ decade). Tin(II)-selective PVC membrane sensor was proposed by Aghaie et al. ${ }^{39}$ They used dibenzo-18-crown-6 (8) (Figure 4) as an ionophore, having a linear working range of $1.0 \times 10^{-6}-1.0 \times 10^{-2} \mathrm{M}$ and a lower detection limit of 8.0 $\times 10^{-7} \mathrm{M}$. In addition, the sensor was used in determination of $\mathrm{Sn}^{2+}$ in various spiked samples. Akl and Abd El-Aziz developed a PVC membrane zinc(II)-selective sensor using 18-crown-6 and dibenzo18crown-6 (8) molecules as ionophores. ${ }^{40}$ The proposed sensors had concentration ranges of $1.0 \times 10^{-5}-$ 
Özbek et al., Org. Commun. (2021) 14:3 228-239

$1.0 \times 10^{-1} \mathrm{M}$. The zinc(II)-selective sensors were used for the determination of zinc in alloy samples. Additionally, the data of zinc(II)-selective sensors were compared with AAS data. They reported that the results of the two methods were consistent. Gupta et al. used dibenzo-18-crown-6 (8) as an ionophore and proposed a nickel(II)-selective potentiometric sensor, ${ }^{41}$ working in a wide concentration range of $1.0 \times 10^{-5}-1.0 \times 10^{-1} \mathrm{M}$. The sensor had a response time of approximately $25 \mathrm{~s}$ and was successfully used to determine $\mathrm{Ni}^{2+}$ ions in Indian brand chocolates.<smiles>C1CCC2OCCOCCOC3CCCCC3OCCOCCOC2C1</smiles>

dicyclohexano-18-crown-6 (6)

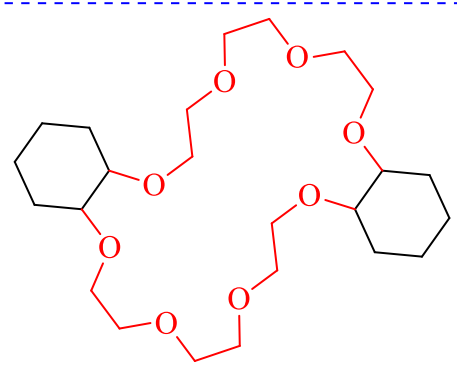

dicyclohexano-24-crown-8 (7)

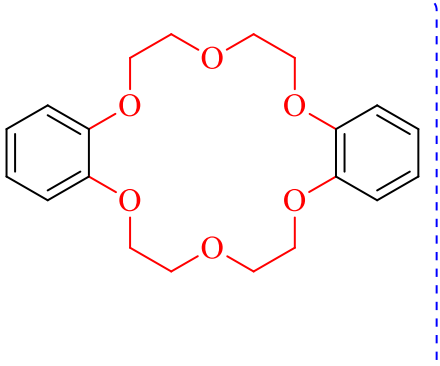

dibenzo-18-crown-6 (8)

Figure 4. Crown ethers 6, 7 and 8 used as ionophores

Shamsipur et al. developed a potentiometric sensor using dibenzo-21-crown-7 (9) (Figure 5) as an ionophore to determine Rubidium ions in tap water samples ${ }^{42}$ which showed a linear response in the concentration range of $5.0 \times 10^{-5}-1.0 \times 10^{-1} \mathrm{M}$ and a $\mathrm{pH}$ range of 3.5-8.0. Potentiometric determination of cadmium(II) ions was performed by Gupta and Kumar using dibenzo-24-crown-8 (10) (Figure 5) as an ionophore. ${ }^{43}$ This sensor displayed a linear response in a concentration range of $3.9 \times 10^{-6}-1.0 \times 10^{-}$ ${ }^{4} \mathrm{M}$ and had a Nernstian slope of $30.0 \pm 1.0 \mathrm{mV} /$ decade. They also stated that the sensor had good selectivity and reproducibility. Another sensor based on dibenzo-24-crown-8 (10) as an ionophore was reported by Gupta et al. for the potentiometric determination of $\mathrm{Zn}^{2+}$ ions. ${ }^{44}$ The sensor had a concentration range of $9.2 \times 10^{-5}-1.0 \times 10^{-1} \mathrm{M}$. It had a $\mathrm{pH}$ working range from 4.8 to 6.2 and a response time of $12 \mathrm{~s}$. In addition, the developed sensor was successfully applied to the determination of $\mathrm{Zn}^{2+}$ ions in wastewater samples. Ganjali et al. developed a potentiometric sensor to determine strontium(II) ions in synthetic water samples. ${ }^{45}$ They used dibenzo-30-crown-10 (11) (Figure 5) as an ionophore. This sensor worked in the concentration range of $1.0 \times 10^{-5}-1.0 \times 10^{-2} \mathrm{M}$ and was shown to be usable in the $\mathrm{pH}$ range of $3.0-10.0$.

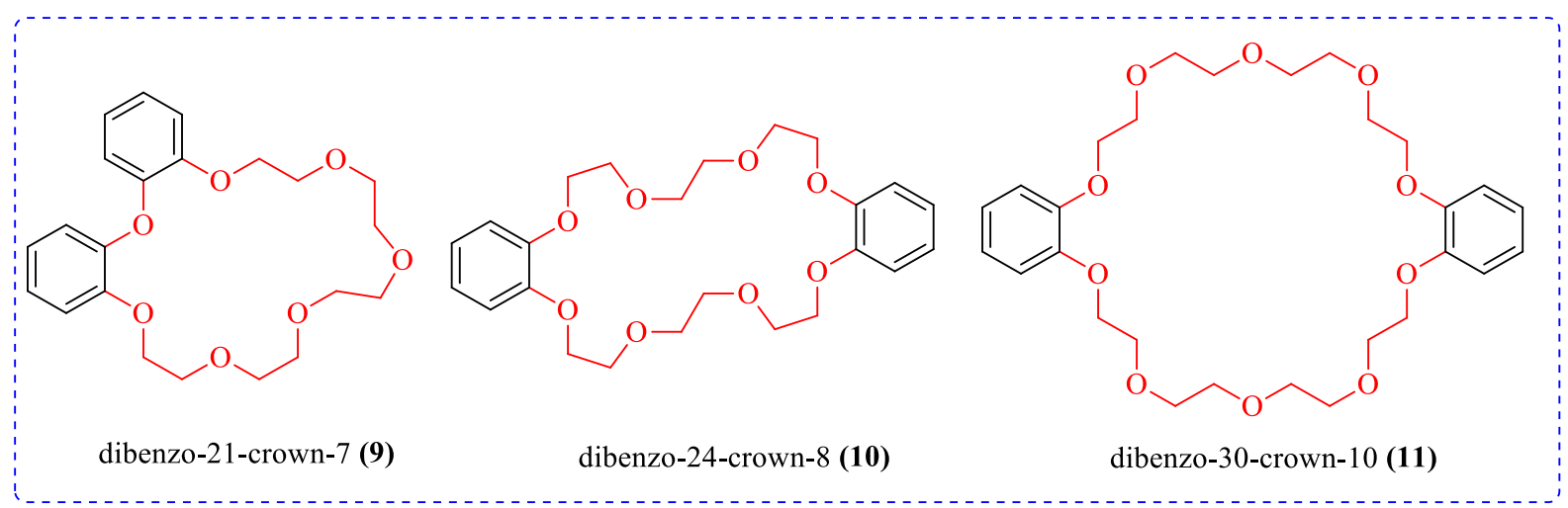

Figure 5. The crown ethers used as ionophores $(\mathbf{9}, \mathbf{1 0}$ and 11)

Sadeghi and Fathi prepared a cesium ion-selective sensor, using 4',4",(5')di-tert-butyldibenzo18-crown-6 (12) (Figure 6) as an ionophore. ${ }^{46}$ The sensor was shown to work in the concentration range of $6.0 \times 10^{-6}-1.0 \times 10^{-2} \mathrm{M}$ and to have a detection limit of $4.0 \times 10^{-6} \mathrm{M}$. The sensor was used in 
The use of crown ethers as sensor material in potentiometry technique

determination of $\mathrm{Cs}^{+}$in spiked tap water samples. The potassium(I)-selective potentiometric sensor was developed by Kemer and Ozdemir.47 They used 4,4'-bis[4"'-phenoxy(15-crown-5)methyl]benzyl molecule (13) (Figure 6) as an ionophore, which had a linear working range of $1.0 \times 10^{-5}-1.0 \times 10^{-1} \mathrm{M}$ and a lower detection limit of $1.0 \times 10^{-7} \mathrm{M}$. Additionally, this sensor was successfully applied in different water samples.

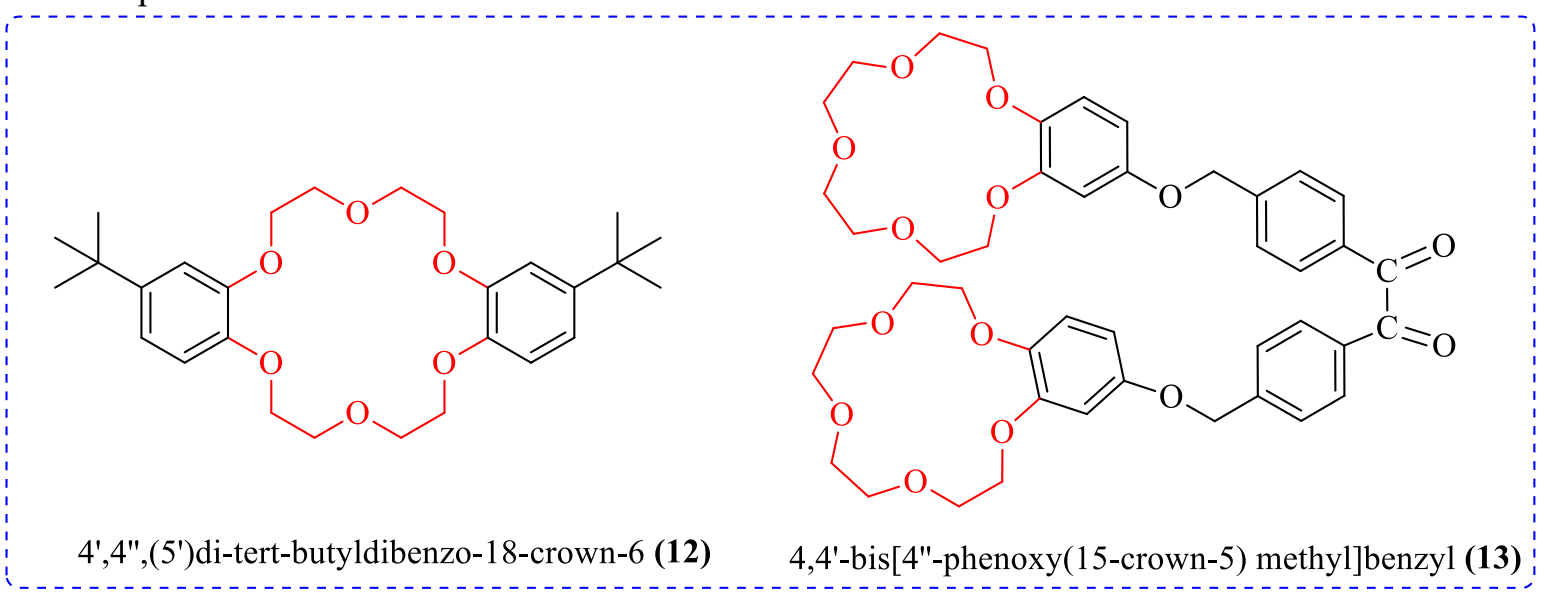

Figure 6. The crown ethers 12 and $\mathbf{1 3}$ used as ionophores

Isildak et al. reported $N, N^{\prime}$-dibenzyl-4,13-diaza-18-crown-6 (14) (Figure 7) as an ionophore in design of PVC membrane zinc(II)-selective sensor. ${ }^{48}$ The proposed sensor worked in a wide concentration range of $1.0 \times 10^{-5}-1.0 \times 10^{-1} \mathrm{M}$ and exhibited a Nernstian behavior $(28.0 \pm 2.0$ $\mathrm{mV} /$ decade). In addition, the sensor had a fast response time of $5 \mathrm{~s}$. Finally, zinc(II)-selective sensor was successfully applied to the potentiometric determination of zinc(II) ions in different water samples and a drug sample. Another work, where $N, N^{\prime}$-dibenzyl-4,13-diaza-18-crown-6 (14) molecule was used as an ionophore. The lead(II)-selective PVC membrane potentiometric sensor was reorted by Gupta et $a l,{ }^{49}$ which had a linear working range of $8.2 \times 10^{-6}-1.0 \times 10^{-1} \mathrm{M}$ with a Nernstian slope of $30.0 \pm 2.0$ $\mathrm{mV} /$ decade. The proposed sensor exhibited a fast response time of about $10 \mathrm{~s}$ and a $\mathrm{pH}$ working range of 2.0-6.8.

A silver(I)-selective polymeric membrane sensor, based on dilaktam crown ether (15) (Figure 7), was developed by Masrournia et al ${ }^{50}$ It exhibited a working concentration range of $1.0 \times 10^{-5}-1.0$ $\times 10^{-1} \mathrm{M}$ and a low detection limit of $6.8 \times 10^{-6} \mathrm{M}$. The electrode was successfully used as an indicator electrode in the potentiometric titration of $\mathrm{Ag}^{+}$against $\mathrm{NaCl}$. A lead(II)-selective $\mathrm{PVC}$ membrane sensor based on 1,4,8,11-tetrathiacyclotetradecane (16) (Figure 7) was reported by Elmosallamy et al ${ }^{51}$ having a concentration range of $1.0 \times 10^{-5}-1.0 \times 10^{-2} \mathrm{M}$ with a Nernstian slope of $29.9 \mathrm{mV} /$ decade. It was successfully used in the determination of $\mathrm{Pb}^{2+}$ ion in the some alloy samples.

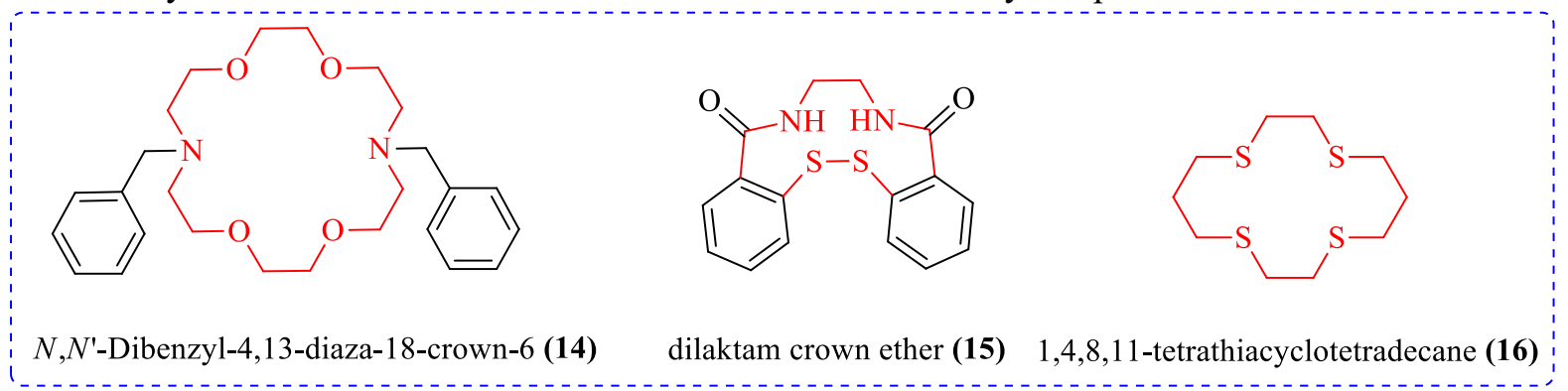

Figure 7. The crown ethers used as ionophores

Golcs et al. developed lead(II)-selective sensors by using acridono-crown ethers (17 and 18) (Figure 8) as ionophores. ${ }^{52}$ They exhibited a Nernstian response for lead(II) ions in the concentration range of $1.0 \times 10^{-4}-1.0 \times 10^{-2} \mathrm{M}$. Furthermore, they reported the use of these lead(II)-selective sensors in the analysis of multicomponent aqueous samples. 
Özbek et al., Org. Commun. (2021) 14:3 228-239

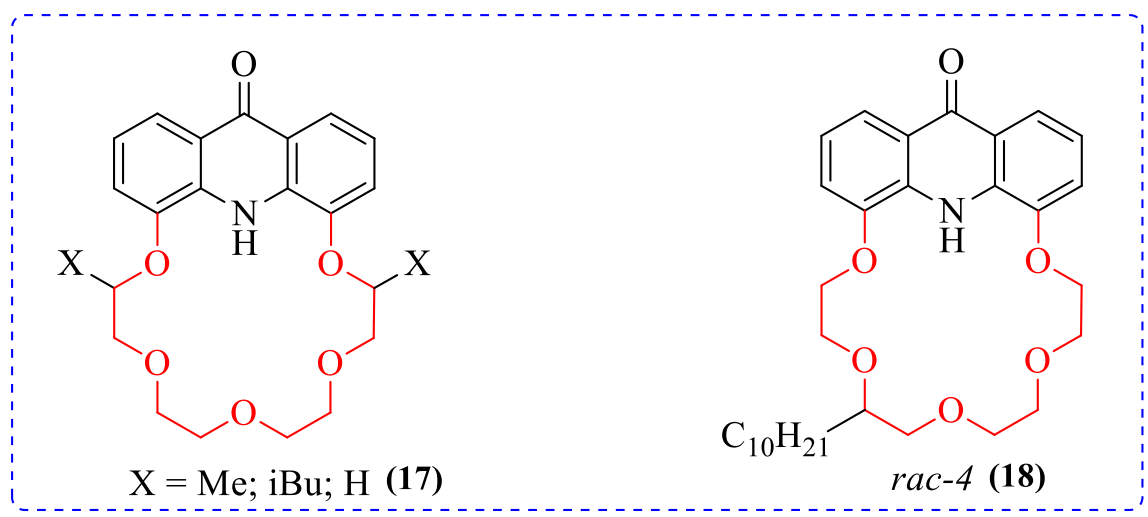

Figure 8. The acridono-crown ethers used as ionophores

Potentiometric characteristics and membrane components of the crown ether-based potentiometric sensors available in the literature are given in Tables 1 and 2. The ionophore (crown ether derivative) ratio in the prepared sensors varies between 0.5 and $9.0 \%$ (Table 1). Besides, it is noteworthy that all the reported sensors have PVC membrane structures.

Table 1. Membrane components of crown ether-based potentiometric sensors in the literature

\begin{tabular}{|c|c|c|c|c|}
\hline \multicolumn{5}{|c|}{ Composition $(\%, w / w)$} \\
\hline Ionophore & Additive & Plasticizer & PVC & Ref. \\
\hline 3.0 & $6.5(\mathrm{OA})$ & 61.5 (DBP) & 29.0 & 27 \\
\hline 4.5 & 4.5 (NaTPB) & 45.5 (DOP) & 45.5 & 28 \\
\hline 4.5 & - & $4.5(\mathrm{DBF})$ & 90.9 & 29 \\
\hline 0.58 & 0.17 (NaTPB) & $59.6(f-\mathrm{PNPE})$ & 39.7 & 30 \\
\hline 5.6 & 3.9 (NaTPB) & $60.5(o-\mathrm{NPOE})$ & 30.0 & 31 \\
\hline 4.0 & - & 20.0 (DOP) & 55.0 & 32 \\
\hline 2.0 & 2.0 (TBATPB) & 67.0 (NPPE) & 29.0 & 33 \\
\hline 9.0 & $3.0(\mathrm{NaTPB})$ & $58.0(o-\mathrm{NPOE})$ & 30.0 & 34 \\
\hline \multicolumn{3}{|c|}{70.0 (ionophore, $o-\mathrm{NPOE}$ and $\mathrm{KTpCIPB}$ ) } & 30.0 & 35 \\
\hline 6.2 & $1.2(\mathrm{NaTPB})$ & 46.3 (DBP) & 46.3 & 36 \\
\hline 6.0 & - & $61.0(o-\mathrm{NPOE})$ & 33.0 & 37 \\
\hline 4.7 & 0.5 & 47.4 (DBBP) & 47.4 & 38 \\
\hline 5.0 & $5.0(\mathrm{OA})$ & 60.0 (AP) & 30.0 & 39 \\
\hline 1.1 & - & 65.9 (DOPP) & 33.0 & 40 \\
\hline 2.4 & 0.2 & 48.7 (TEHP) & 48.7 & 41 \\
\hline 6.5 & $2.7(\mathrm{NaTPB})$ & $56.9(o-\mathrm{NPOE})$ & 33.9 & 42 \\
\hline 4.8 & - & $47.6(\mathrm{DBBP})$ & 47.6 & 43 \\
\hline 3.2 & 0.64 & 32.0 (DOP) & 64.1 & 44 \\
\hline 5.0 & $10.0(\mathrm{OA})$ & 55.0 (BA) & 30.0 & 45 \\
\hline 8.0 & $1.0(\mathrm{NaTPB})$ & 58.0 (DOP) & 33.0 & 46 \\
\hline 4.0 & $1.0(\mathrm{NaTPB})$ & $55.0(\mathrm{DOS})$ & 40.0 & 47 \\
\hline 4.0 & $1.0(\mathrm{KTpClPB})$ & 62.0 (BEHS) & 33.0 & 48 \\
\hline 3.83 & 0.32 (NaTPB) & 31.9 (DBP) & 63.9 & 49 \\
\hline 4.0 & - & 63.0 (DOP) & 33.0 & 50 \\
\hline 1.0 & - & $66.0(o-\mathrm{NPOE})$ & 33.0 & 52 \\
\hline
\end{tabular}


The use of crown ethers as sensor material in potentiometry technique

Table 2. Potentiometric characteristics of crown ethers-based potentiometric sensors

\begin{tabular}{|c|c|c|c|c|c|c|c|c|}
\hline Ionophore & Ion & $\begin{array}{l}\text { Concentration } \\
\text { range }(\mathbf{M})\end{array}$ & $\begin{array}{l}\text { Limit of } \\
\text { detection } \\
\text { (M) }\end{array}$ & $\begin{array}{c}\text { pH } \\
\text { working } \\
\text { range } \\
\end{array}$ & $\begin{array}{l}\text { Response } \\
\text { time (s) }\end{array}$ & $\begin{array}{c}\text { Slope } \\
\text { (mV/decade } \\
\text { ) }\end{array}$ & $\begin{array}{c}\text { Life } \\
\text { time } \\
\text { (month) }\end{array}$ & Ref. \\
\hline 12-crown-4 (1) & $\mathrm{Al}^{3+}$ & $\begin{array}{l}1.0 \times 10^{-6}- \\
1.0 \times 10^{-1}\end{array}$ & $5.5 \times 10^{-7}$ & $2.0-7.8$ & 15 & - & 2 & 27 \\
\hline 12-crown-4 (1) & $\mathrm{Zn}^{2+}$ & $\begin{array}{c}7.0 \times 10^{-5}- \\
1.0 \times 10^{-1}\end{array}$ & - & $2.8-5.5$ & $<10$ & $29.5 \pm 1.0$ & 3 & 28 \\
\hline 12-crown-4 (1) & $\mathrm{Cu}^{2+}$ & $\begin{array}{c}1.78 \times 10^{-5}- \\
1.0 \times 10^{-1}\end{array}$ & $1.78 \times 10^{-5}$ & $3.0-6.0$ & $<30$ & 50.0 & 6 & 29 \\
\hline 15-crown-5 (2) & $\mathrm{Pb}^{2+}$ & $\begin{array}{l}1.0 \times 10^{-6}- \\
1.0 \times 10^{-2}\end{array}$ & $2.0 \times 10^{-7}$ & - & $<2$ & $31.14 \pm 0.94$ & 6 & 30 \\
\hline 15-crown-5 (2) & $\mathrm{Ag}^{+}$ & $\begin{array}{c}1.0 \times 10^{-7}- \\
1.0 \times 10^{-1}\end{array}$ & $8.09 \times 10^{-7}$ & $3.0-8.0$ & $<10$ & 58.9 & 2 & 31 \\
\hline benzo-18-crown-6 (3) & $\mathrm{Pb}^{2+}$ & $\begin{array}{c}1.0 \times 10^{-5}- \\
1.0 \times 10^{-1}\end{array}$ & $5.0 \times 10^{-6}$ & $1.5-5.0$ & 30 & 28.8 & 3 & 32 \\
\hline benzo-18-crown-6 (3) & $\mathrm{Fe}^{3+}$ & $\begin{array}{c}1.0 \times 10^{-6}- \\
1.0 \times 10^{-1}\end{array}$ & - & - & 30 & $57.0 \pm 1.0$ & 2 & 33 \\
\hline naphto-9-crown-3 (4) & $\mathrm{Be}^{2+}$ & $\begin{array}{c}8.0 \times 10^{-6}- \\
1.0 \times 10^{-1}\end{array}$ & $6.0 \times 10^{-6}$ & $3.5-9.0$ & $<15$ & 29.5 & - & 34 \\
\hline $\begin{array}{l}\text { 6,6-dibenzyl-14- } \\
\text { crown-4 (5) }\end{array}$ & $\mathrm{Li}^{+}$ & $\begin{array}{c}1.0 \times 10^{-4}- \\
2.0 \times 10^{-1}\end{array}$ & $3.0 \times 10^{-5}$ & $4.0-8.0$ & $<100$ & $58.5 \pm 1.0$ & 4 & 35 \\
\hline $\begin{array}{l}\text { dicyclohexano-18- } \\
\text { crown-6 (6) }\end{array}$ & $\mathrm{Cd}^{2+}$ & $\begin{array}{l}2.1 \times 10^{-5}- \\
1.0 \times 10^{-1}\end{array}$ & - & $1.9-7.0$ & 17 & $29.0 \pm 1.0$ & 6 & 36 \\
\hline $\begin{array}{l}\text { dicyclohexano-18- } \\
\text { crown-6 (6) }\end{array}$ & $\mathrm{La}^{3+}$ & $\begin{array}{c}1.0 \times 10^{-6}- \\
1.0 \times 10^{-1}\end{array}$ & $5.0 \times 10^{-7}$ & $4.0-9.0$ & $<30$ & 19.0 & 5 & 37 \\
\hline $\begin{array}{l}\text { dicyclohexano-24- } \\
\text { crown-8 (7) }\end{array}$ & $\mathrm{Cd}^{2+}$ & $\begin{array}{c}3.0 \times 10^{-5}- \\
1.0 \times 10^{-1}\end{array}$ & - & $2.0-5.4$ & 23 & $30.0 \pm 1.0$ & 6 & 38 \\
\hline $\begin{array}{c}\text { dibenzo- } 18 \text {-crown-6 } \\
\text { (8) }\end{array}$ & $\mathrm{Sn}^{2+}$ & $\begin{array}{c}1.0 \times 10^{-6}- \\
1.0 \times 10^{-2}\end{array}$ & $8.0 \times 10^{-7}$ & - & $<15$ & $27.5 \pm 0.6$ & 3 & 39 \\
\hline $\begin{array}{c}\text { dibenzo- } 18 \text {-crown-6 } \\
\text { (8) }\end{array}$ & $\mathrm{Zn}^{2+}$ & $\begin{array}{c}1.0 \times 10^{-5}- \\
1.0 \times 10^{-1}\end{array}$ & $1.5 \times 10^{-5}$ & $4.0-8.0$ & $\prec 15$ & 30.0 & 2 & 40 \\
\hline $\begin{array}{c}\text { dibenzo- } 18 \text {-crown-6 } \\
\text { (8) }\end{array}$ & $\mathrm{Ni}^{2+}$ & $\begin{array}{c}1.0 \times 10^{-5}- \\
1.0 \times 10^{-1}\end{array}$ & - & $2.6-6.8$ & $<25$ & 29.5 & 4 & 41 \\
\hline $\begin{array}{c}\text { dibenzo- } 21 \text {-crown-7 } \\
\text { (9) }\end{array}$ & $\mathrm{Rb}^{2+}$ & $\begin{array}{c}5.0 \times 10^{-5}- \\
1.0 \times 10^{-1}\end{array}$ & $1.5 \times 10^{-6}$ & $3.5-8.0$ & $<40$ & 57.8 & $\sim 2$ & 42 \\
\hline $\begin{array}{c}\text { dibenzo-24-crown-8 } \\
\text { (10) }\end{array}$ & $\mathrm{Cd}^{2+}$ & $\begin{array}{c}3.9 \times 10^{-6}-1.0 \\
\times 10^{-1}\end{array}$ & - & $3.2-7.5$ & 25 & $30.0 \pm 1.0$ & 5 & 43 \\
\hline $\begin{array}{c}\text { dibenzo-24-crown-8 } \\
\mathbf{( 1 0 )}\end{array}$ & $\mathrm{Zn}^{2+}$ & $\begin{array}{l}9.2 \times 10^{-5}- \\
1.0 \times 10^{-1}\end{array}$ & $2.0 \times 10^{-7}$ & $4.8-6.2$ & 12 & $29.0 \pm 0.5$ & 4 & 44 \\
\hline $\begin{array}{c}\text { dibenzo-30-crown-10 } \\
\text { (11) }\end{array}$ & $\mathrm{Sr}^{2+}$ & $\begin{array}{c}1.0 \times 10^{-5}- \\
1.0 \times 10^{-1}\end{array}$ & $5.0 \times 10^{-6}$ & $3.0-10.0$ & $<10$ & $29.2 \pm 0.3$ & 4 & 45 \\
\hline $\begin{array}{l}\text { 4',4",(5')di-tert- } \\
\text { butyldibenzo-18- } \\
\text { crown-6 (12) }\end{array}$ & $\mathrm{Cs}^{+}$ & $\begin{array}{c}6.0 \times 10^{-6}- \\
1.0 \times 10^{-1}\end{array}$ & $4.0 \times 10^{-6}$ & $3.0-9.5$ & 10 & $57.0 \pm 1.8$ & 3 & 46 \\
\hline $\begin{array}{l}\text { 4,4'-bis[4"-phenoxy } \\
\text { (15-crown-5) } \\
\text { methyl]benzyl (13) }\end{array}$ & $\mathrm{K}^{+}$ & $\begin{array}{l}1.0 \times 10^{-5}- \\
1.5 \times 10^{-1}\end{array}$ & $1.0 \times 10^{-7}$ & $5.0-7.0$ & $<10$ & $55.0 \pm 15$ & 2 & 47 \\
\hline $\begin{array}{c}N, N^{\prime} \text {-Dibenzyl-4,13- } \\
\text { diaza-18-crown-6 (14) }\end{array}$ & $\mathrm{Zn}^{2+}$ & $\begin{array}{c}1.0 \times 10^{-5}- \\
1.0 \times 10^{-1}\end{array}$ & $1.17 \times 10^{-6}$ & $4.0-11.0$ & 5 & $28.0 \pm 2.0$ & - & 48 \\
\hline $\begin{array}{c}N, N^{\prime} \text {-Dibenzyl-4,13- } \\
\text { diaza-18-crown-6 (14) }\end{array}$ & $\mathrm{Pb}^{2+}$ & $\begin{array}{l}8.2 \times 10^{-6}- \\
1.0 \times 10^{-1}\end{array}$ & $>8.2 \times 10^{-6}$ & $2.0-6.8$ & 10 & $30.0 \pm 0.1$ & 3 & 49 \\
\hline $\begin{array}{l}\text { dilaktam crown ether } \\
\text { (15) }\end{array}$ & $\mathrm{Ag}^{+}$ & $\begin{array}{c}1.0 \times 10^{-5}- \\
1.0 \times 10^{-1}\end{array}$ & $6.8 \times 10^{-6}$ & $5.1-7.2$ & 20 & $59.8 \pm 0.2$ & 2.5 & 50 \\
\hline $\begin{array}{l}\text { 1,4,8,11-tetrathiacyclo } \\
\text { tetradecane } \mathbf{( 1 6 )}\end{array}$ & $\mathrm{Pb}^{2+}$ & $\begin{array}{c}1.0 \times 10^{-5}- \\
1.0 \times 10^{-2}\end{array}$ & $2.2 \times 10^{-6}$ & $3.0-6.5$ & 20 & 29.9 & 3 & 51 \\
\hline $\begin{array}{c}\text { acridono-crown ether } \\
(\mathbf{1 7} \text { and } \mathbf{1 8})\end{array}$ & $\mathrm{Pb}^{2+}$ & $\begin{array}{c}1.0 \times 10^{-4}- \\
1.0 \times 10^{-2}\end{array}$ & $7.9 \times 10^{-6}$ & $4.0-7.0$ & 5 & 26.9 & 3 & 52 \\
\hline
\end{tabular}




\section{Applications of Crown Ether-Based Potentiometric Sensors}

The real sample applications and recovery of PVC membrane crown ether-based potentiometric sensors reported in the literature are given in Table 3. The proposed crown ether-based sensors demonstrated very high recoveries in different real sample analyses. In addition, the developed potentiometric sensors showed compatible results with other analytical methods such as AAS, AES, pulse polargraphy and Inductively Coupled Plasma spectroscopy (ICP).

Table 3. Real sample applications of PVC membrane crown ether-based sensors in the literature

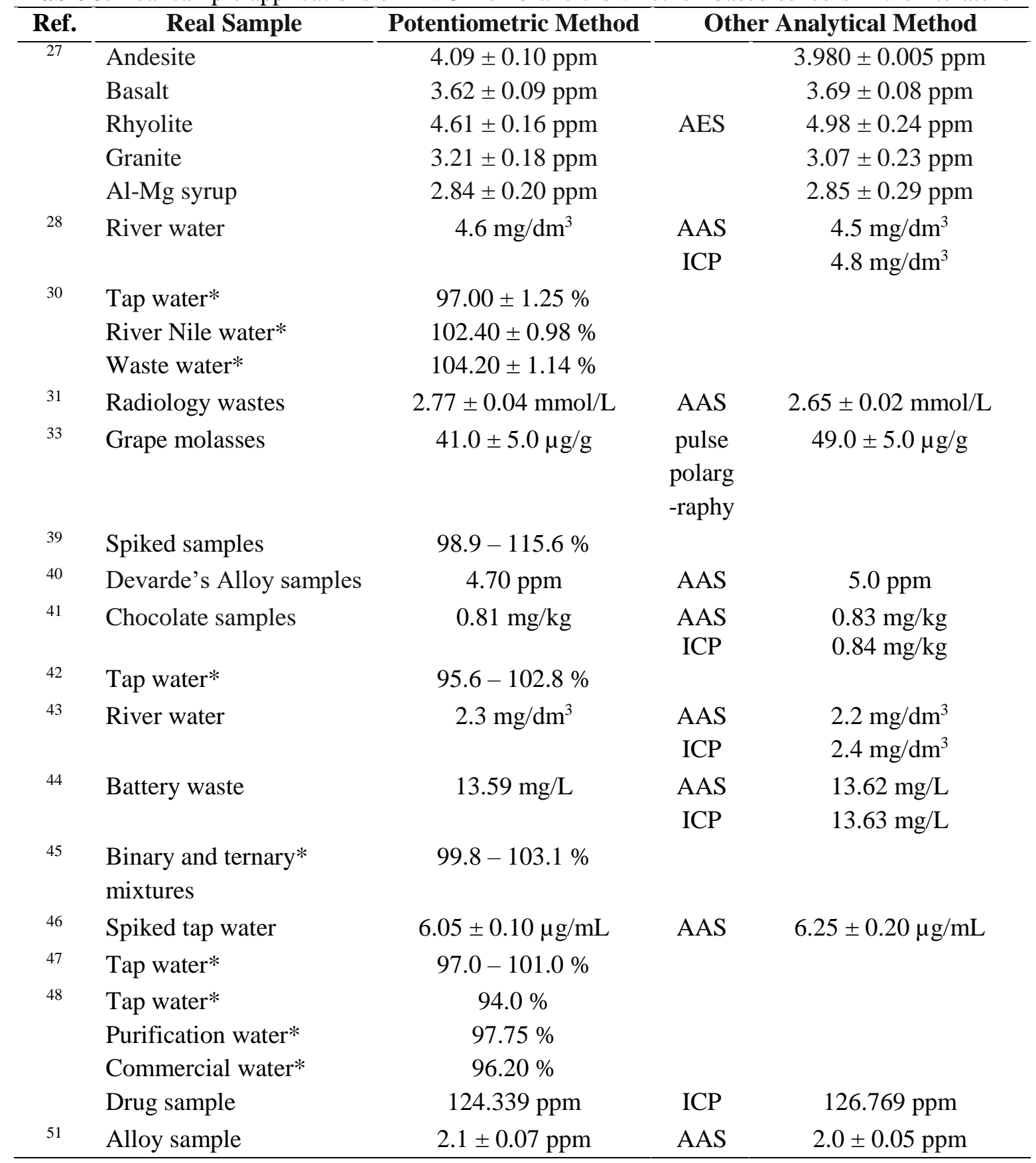

*Standard addition method

Crown ethers were reported to be used as sensors in voltammetry technique (Table 4). 
The use of crown ethers as sensor material in potentiometry technique

Table 4. Some crown-ether derivatives used in voltammetry technique

\begin{tabular}{cccccc} 
Crown ether & Ion & $\begin{array}{c}\text { Concentration } \\
\text { range }\end{array}$ & $\begin{array}{c}\text { Limit of } \\
\text { detection }\end{array}$ & Real sample & Ref \\
\hline $\begin{array}{c}\text { 12-crown-4 (1) } \\
\text { dicyclohexyl-18- } \\
\text { crown-6 }\end{array}$ & $\mathrm{Hg}^{2+}$ & $5.0-110.0 \mu \mathrm{g} / \mathrm{ml}$ & $0.25 \mu \mathrm{g} / \mathrm{ml}$ & $\begin{array}{c}\text { blood, urine, } \\
\text { tap water }\end{array}$ & 53 \\
$\begin{array}{c}\mathrm{Tl}^{+} \\
\text {benzo-15-crown-5 }\end{array}$ & $\mathrm{Cu}^{2+}$ & $1.0-100.0 \mathrm{ppb}$ & $0.05 \mathrm{ppm}$ & $\begin{array}{c}\text { alcoholic } \\
\text { beverages }\end{array}$ & 55 \\
$\begin{array}{c}\text { diaza-18-crown-6 } \\
\mathbf{( 1 4 )}\end{array}$ & $\mathrm{Pb}^{2+}$ & $10.0-50.0 \mu \mathrm{g} / \mathrm{L}$ & $0.09 \mu \mathrm{g} / \mathrm{L}$ & River water & 56 \\
$\begin{array}{c}\text { 18-crown-6 } \\
\mathrm{Hg}^{2+}\end{array}$ & $\begin{array}{c}1.0 \times 10^{-5}- \\
6.0 \times 10^{-6} \mathrm{M}\end{array}$ & $2.0 \times 10^{-7} \mathrm{M}$ & Not reported & 57 \\
\hline
\end{tabular}

\section{Conclusion}

Macrocyclic molecules are used extensively by researchers in many fields due to their unique properties. These molecules, containing multiple donor atoms such as $\mathrm{N}, \mathrm{O}$, and $\mathrm{S}$ in their structures, can be considered sensor materials (ionophore) and exhibit selectivity towards various ions. ${ }^{25}$ This review paper describes the use of crown ethers as ionophores in potentiometric ion-selective electrodes. Crown ethers, an important group of macrocycles, have been subject to extensive research in developing potentiometry-based sensors. They can bind to cations due to their structures with suitable cavities. Therefore, crown ethers are highly attractive molecules for sensor studies, and have been included as ionophores in many studies. As a conclusion, crown ethers are considered very attractive molecules as ion-selective electrodes.

\section{ORCID}

Oguz Özbek: 0000-0001-5185-9681

Ömer Isildak: 0000-0003-4690-4323

Meliha Burcu Gürdere: 0000-0003-4285-5528

Alper Cetin: 0000-0002-6093-9605

\section{References}

[1] Kralj, M.; Tušek-Božić, L.; Frkanec, L. Biomedical potentials of crown ethers: prospective antitumor agents. ChemMedChem 2008, 3(10), 1478-1492.

[2] Pedersen, C.J. Cyclic polyethers and their complexes with metal salts. J. Am. Chem. Soc. 1967, 80(10), 2495-2496.

[3] Pedersen, C.J. Cyclic polyethers and their complexes with metal salts. J. Am. Chem. Soc. 1967, 89(26), 7017-7036.

[4] Schneider, T.; Brüssow, N.; Yuvanc, A.; Budisa, N. Synthesis of new aza-and thia-crown ether based amino acids. ChemistrySelect 2020, 5(9), 2854-2857.

[5] Mohammadzadeh Kakhki, R. Application of crown ethers as stationary phase in the chromatographic methods. J. Incl. Phenom. Macrocycl. Chem. 2013, 75, 11-22.

[6] Chehardoli, G.; Bahmani A. The role of crown ethers in drug delivery. Supramol. Chem. 2019, 31(4), 221238.

[7] Rounaghi, G.; Zade Kakhki R. M. Thermodynamic study of complex formation between dibenzo-18crown-6 and $\mathrm{UO}_{2}{ }^{2+}$ cation in different non-aqueous binary solutions. J. Incl. Phenom. Macrocycl. Chem. 2009, 63, 117.

[8] Fathalla, M. Synthesis and characterization of a porphyrin-crown ether conjugate as a potential intermediate for drug delivery application. J. Porphyr. Phthalocyanines 2021, 25(2), 95-101. 
Özbek et al., Org. Commun. (2021) 14:3 228-239

[9] Alexandratos, S.D.; Stine, C.L. Synthesis of ion-selective polymer-supported crown ethers: a review. React. Funct. Polym. 2004, 60, 3-16.

[10] Rounaghi, G.; Zavar, M. H.; Zade Kakhki, R. M. Thermodynamic behavior of complexation process between dibenzo-18-crown- 6 and $\mathrm{K}^{+}, \mathrm{Ag}^{+}, \mathrm{NH}_{4}{ }^{+}$, and $\mathrm{Hg}^{2+}$ cations in ethylacetate-dimethylformamide binary media. Russ. J. Coord. Chem. 2008, 34(3), 167-171.

[11] Hasanova, U.A.; Ramazanov, M.A.; Maharramov, A.M.; Gakhramanova, Z.; Hajiyeva, S.F.; Vezirova, L.; Eyvazova, G.M.; Hajiyeva, F.V.; Huseynova, P.; Agamaliyev, Z. The functionalization of magnetite nanoparticles by hydroxyl substituted diazacrown ether, able to mimic natural siderophores, and investigation of their antimicrobial activity. J. Incl. Phenom. Macrocycl. Chem. 2016, 86, $19-25$.

[12] Marjanovic, M.; Kralj, M.; Supek, F.; Frkanec, L.; Piantanida, I.; Smuc, T.; Tusek-Bozic, L. Antitumor potential of crown ethers: Structure-activity relationships, cell cycle disturbances, and cell death studies of a series of ionophores. J. Med. Chem. 2007, 50, 1007-1018.

[13] Hayvalı, Z.; Güler, H.; Öğütcü, H.; Sarı N. Novel bis-crown ethers and their sodium complexes as antimicrobial agent: synthesis and spectroscopic characterizations. Med. Chem. Res. 2014, 23, 3652-3661.

[14] Famaey, J.P.; Whitehouse, M.W. About some possible anti-inflammatory properties of various membrane permeant agents. Agents Action. 1975, 5, 133-136.

[15] Zasukhina, G.D.; Vasil'eva, I.M.; Vedernikov, A.I.; Gromov, S.P.; Alfimov, M.V.; Antimutagenic characteristics of new diazacrown compounds with N-carboxyalkyl substitutes. Bull. Exp. Biol. Med. 2006, 141, 331-333.

[16] Isildak, Ö.; Özbek O. Application of potentiometric sensors in real samples. Crit. Rev. Anal Chem. 2021, 51(3) 218-231.

[17] Özbek, O.; Berkel, C.; Isildak Ö. Applications of potentiometric sensors for the determination of drug molecules in biological samples. Crit. Rev. Anal Chem. 2020, 1-12. doi.10.1080/10408347.2020.1825065

[18] Isildak, Ö.; Özbek, O.; Gürdere, M. B. Development of chromium(III)-selective potentiometric sensor by using synthesized pyrazole derivative as an ionophore in PVC matrix and its applications. J. Anal. Test. 2020, 4(4), 273-280.

[19] Isildak, Ö.; Özbek, O.; Yigit, K. M. A bromide-selective PVC membrane potentiometric sensor. Bulg. Chem. Commun. 2020, 52(4), 448-452.

[20] Suman, S.; Singh R. Iodide-selective PVC membrane electrode based on copper complex of 2acetylthiophene semicarbazone as carrier. Anal. Chem. Lett. 2020, 10(3), 357-365.

[21] Işsldak, Ö.; Deligönül, N.; Özbek, O. A novel silver(I)-selective PVC membrane sensor and its potentiometric applications. Turk. J. Chem. 2019, 43(4), 1149-1158.

[22] Iş̧1dak, Ö.; Özbek, O. Silver (I)-selective PVC membrane potentiometric sensor based on 5, 10, 15, 20tetra (4-pyridyl)-21H,23H-porphine and potentiometric applications. J. Chem. Sci. 2020, 132(1), 1-8.

[23] Özbek, O.; Isildak, Ö.; Berkel, C. The use of porphyrins in potentiometric sensors as ionophores. J. Incl. Phenom. Macrocycl. Chem. 2020, 98(1-2), 1-9.

[24] Topcu, C.; Isildak I. Novel micro flow injection analysis system for the potentiometric determination of tetraborate ions in environmental samples. Anal. Lett. 2021, 5, 854-866.

[25] Özbek, O.; Isildak, Ö. Polymer-based cadmium (II)-selective potentiometric sensors for the analysis of $\mathrm{Cd}^{2+}$ in different environmental samples. Int. J. Environ. Anal. Chem. 2021, 1-14. doi.10.1080/03067319.2021.1877283

[26] Özbek, O.; Isildak, Ö.; Gürdere, M. B.; Berkel, C. Cadmium(II)-selective potentiometric sensor based on synthesised (E)-2-benzylidenehydrazinecarbothioamide for the determination of $\mathrm{Cd}^{2+}$ in different environmental samples. I Int. J. Environ. Anal. Chem. 2020, 1-16. https://doi.org/10.1080/03067319.2020.1817427

[27] Esmaelpourfarkhani, M.; Rounaghi, G. H.; Arbab Zavar, M. H. Construction of a new aluminum(III) cation selective electrode based on 12-crown-4 as an ionophore. J. Braz. Chem. Soc. 2015, 26(5), 963-969.

[28] Gupta, V. K. A PVC-based 12-crown-4 membrane potentiometric sensor for zinc(II) ions. Sens. Actuators B Chem. 1999, 55(2-3), 195-200.

[29] Kumar, D. M.; Suresh, J.; Neeraj, J.; Poonam, B. A PVC-based crown ether membrane sensor for $\mathrm{Cu}^{2+}$. Res. J. Chem. Environ. 2013, 17(9), 82-85.

[30] Khaled, E.; Kamel, M. S.; Hassan, H. N. A. Novel multi walled carbon nanotubes/crown ether based disposable sensors for determination of lead in water samples. Anal. Chem. Lett. 2015, 5(6), 329-337.

[31] Karimian, F.; Rounaghi, G. H.; Arbab-Zavar, M. H. Construction of a PVC based 15-crown-5 electrochemical sensor for Ag (I) cation. Chin. Chem. Lett. 2014, 25(5), 809-814.

[32] Ghorbani, S.; Rounaghi, G. H.; Tarahomi, S.; Mohajeri, M. Lead(II)-selective coated graphite electrode based on benzo-18-crown-6. Asian J. Chem. 2013, 25(2), 905-908. 
The use of crown ethers as sensor material in potentiometry technique

[33] Ekmekci, G.; Uzun, D.; Somer, G.; Kalayc1, Ş. A novel iron (III) selective membrane electrode based on benzo-18-crown-6 crown ether and its applications. J. Membr. Sci. 2007, 288(1-2), 36-40.

[34] Ganjali, M. R.; Daftari, A.; Faal-Rastegar, M.; Moghimi, A. Novel potentiometric sensor for monitoring beryllium based on naphto-9-crown-3. Anal. Sci. 2003, 19(3), 353-356.

[35] Govindan, R.; Alamelu, D.; Vittal Rao, T. V.; Bamankar, Y. R.; Mukarjee, S. K.; Parida, S. C.; Joshi, A. R. Determination of lithium in organic matrix using coated wire lithium ion selective electrode. Indian $J$. Adv. Chem. Sci. 2014, 2, 89-94.

[36] Gupta, V. K.; Chandra, S.; Mangla, R. Dicyclohexano-18-crown-6 as active material in PVC matrix membrane for the fabrication of cadmium selective potentiometric sensor. Electrochim. Acta 2002, 47(10), $1579-1586$.

[37] Mittal, S. K.; Kumar, S. A.; Sharma, H. K. PVC-based dicyclohexano-18-crown-6 sensor for La (III) ions. Talanta 2004, 62(4), 801-805.

[38] Gupta, V. K.; Jain, A. K.; Kumar, P. PVC-based membranes of dicyclohexano-24-crown-8 as Cd(II) selective sensor. Electrochim. Acta 2006, 52(2), 736-741.

[39] Aghaie, H.; Giahi, M.; Monajjemi, M.; Arvand, M.; Nafissi, G. H.; Aghaie, M. Tin (II)-selective membrane potentiometric sensor using a crown ether as neutral carrier. Sens. Actuators B Chem. 2005, 107(2), 756761.

[40] Akl, M. A.; Abd El-Aziz, M. H. Polyvinyl chloride-based 18-crown-6, dibenzo18-crown-6 and calix-[6]arene zinc (II)-potentiometric sensors. Arab. J. Chem. 2016, 9, 878-888.

[41] Gupta, V. K.; Goyal, R. N.; Agarwal, S.; Kumar, P.; Bachheti, N. Nickel (II)-selective sensor based on dibenzo-18-crown-6 in PVC matrix. Talanta 2007, 71(2), 795-800.

[42] Shamsipur, M.; Alizadeh, K.; Hosseini, M.; Mousavi, M. F.; Ganjali, M. R. PVC membrane and coated graphite potentiometric sensors based on dibenzo-21-crown-7 for selective determination of rubidium ions. Anal. Lett. 2005, 38(4), 573-588.

[43] Gupta, V. K.; Kumar P. Cadmium(II)-selective sensors based on dibenzo-24-crown-8 in PVC matrix. Anal. Chim. Acta 1999, 389(1-3), 205-212.

[44] Gupta, V. K.; Al Khayat, M.; Minocha, A. K.; Kumar, P. Zinc (II)-selective sensors based on dibenzo-24crown-8 in PVC matrix. Anal. Chim. Acta 2005, 532(2), 153-158.

[45] Ganjali, M. R.; Kiani, R.; Yousefi, M.; Faal-Rastegar, M. Novel potentiometric strontium membrane sensor based on dibenzo-30-crown-10. Anal. Lett. 2003, 36(10), 2123-2137.

[46] Sadeghi, S.; Fathi F. Polymeric membrane coated graphite cesium selective electrode based on $4^{\prime}, 4^{\prime \prime}\left(5^{\prime}\right) \mathrm{di}-$ tert-butyl di-benzo-18-crown-6. J. Incl. Phenom. Macrocycl. Chem. 2010, 67(1-2), 91-98.

[47] Kemer, B.; Ozdemir, M. Potentiometric utility of the new solid-state sensor based on crowned ionophore for the determination of $\mathrm{K}^{+}$. Turk. J. Chem. 2008, 32, 521-528.

[48] Isildak, Ö.; Özbek, O.; Yigit, K. M. Zinc (II)-selective PVC membrane potentiometric sensor for analysis of $\mathrm{Zn}^{2+}$ in drug sample and different environmental samples. Int. J. Environ. Anal. Chem. 2019, 1-11. doi.10.1080/03067319.2019.1691542

[49] Gupta, V. K.; Jain, A. K.; Kumar, P. PVC-based membranes of N,N'-dibenzyl-1,4,10,13-tetraoxa-7,16diazacyclooctadecane as $\mathrm{Pb}$ (II)-selective sensor. Sens. Actuators B Chem. 2006, 120(1), 259-265.

[50] Masrournia, M.; Zamani. H. A.; Mohamadzadeh, H.; Seyedi, S. M.; Ganjali, M. R.; Eshghi, H. A silver (I) PVC-membrane sensor based on synthesized dilaktam crown ether. J. Chil. Chem. Soc. 2009, 54(1), $63-$ 67.

[51] Elmosallamy, M. A. F.; Fathy, A. M.; Ghoneim, A. K. Lead(II) potentiometric sensor based on 1,4,8,11tetrathiacyclotetradecane neutral carrier and lipophilic additives. Electroanal. 2008, 20, 1241-1245.

[52] Golcs, Á.; Horváth, V.; Huszthy, P.; Tóth, T. Fast potentiometric analysis of lead in aqueous medium under competitive conditions using an acridono-crown ether neutral ionophore. Sensors 2018, 18(5), 1407.

[53] Hassan, R.Y.; Kamel, M.S.; Hassan, H.N.; Khaled, E. Voltammetric determination of mercury in biological samples using crown ether/multiwalled carbon nanotube-based sensor. J. Electroanal. Chem. 2015, 759, 101-106.

[54] Cheraghi, S.; Taher, M. A.; Fazelirad, H. Voltammetric sensing of thallium at a carbon paste electrode modified with a crown ether. Microchim. Acta 2013, 180, 1157-1163.

[55] Ijeri, V.; Srivastava, A. Voltammetric determination of copper at chemically modified electrodes based on crown ethers. Fresenius J. Anal. Chem. 2000, 367, 373-377. 
Özbek et al., Org. Commun. (2021) 14:3 228-239

[56] Segura, R.; Díaz, K.; Pizarro, J.; Placencio, A.; Tapia, D.; Fajardo, Á. Anodic stripping voltammetric determination of lead using a chemically modified electrode based on AZA crown ether. J. Chil. Chem. Soc. 2017, 62(4), 3726-3730.

[57] Wang, J.; Bonakdar, M. Preconcentration and voltammetric measurement of mercury with a crown-ether modified carbon-paste electrode. Talanta 1988, 35(4), 277-280.

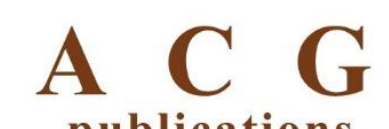

publications

(C) 2021 ACG Publications 